\title{
Terapia de produção audiovisual associada a estimulação por corrente contínua melhora nomeação em paciente com afasia de Broca e doença de Parkinson
}

\section{Audiovisual production therapy associated with transcranial current stimulation improves naming in a patient with Broca's aphasia and} Parkinson's disease

\author{
Cláudia Aparecida Pietrobon ${ }^{1}$ (D), Ricardo Marcio Garcia Rocha' (1D), Juliana Silva de Deus ${ }^{1}$ (D), \\ Marcos Felipe Rodrigues de Lima' ${ }^{1}$, Beatriz Araújo Cavendish ${ }^{1}$ (D), Luciano Grüdtner Buratto ${ }^{10}$
}

\section{RESUMO}

A doença de Parkinson (DP) é uma doença neurodegenerativa, caracterizada por disfunções motoras e não motoras. Pacientes com DP também podem apresentar problemas de linguagem, incluindo deficit em tarefas de nomeação. Dificuldade em tarefas de nomeação é uma característica importante da afasia de Broca, transtorno de linguagem associado a lesões pós-acidente vascular cerebral (AVC) no córtex pré-frontal inferior esquerdo (área de Broca). Aqui, apresenta-se o caso de um paciente de 79 anos diagnosticado com DP (estágio 4 na escala de Hoehn e Yahr) e afasia crônica não fluente pós-AVC, com deficit de nomeação severos. O paciente foi tratado com uma nova combinação de terapia audiovisual de produção e estimulação transcraniana por corrente contínua (ETCC), técnica neuromodulatória não invasiva, que tem sido cada vez mais adotada para potencializar terapias fonoaudiológicas. ETCC anodal ( $2 \mathrm{~mA})$ foi aplicada sobre o córtex préfrontal inferior esquerdo (F7 no sistema 10/20), durante nove sessões de 20 minutos, ao longo de duas semanas, enquanto o paciente tentava nomear imagens de objetos comuns com o auxílio de vídeos curtos mostrando uma boca articulando os sons do nome do objeto (pista audiovisual). Observou-se aumento significativo nos escores de nomeação entre o pré e o pós-tratamento, tanto para imagens treinadas, quanto para não treinadas, mas fonemicamente similares (generalização). Os resultados apresentaram indícios iniciais de que terapia audiovisual de produção associada à ETCC anodal sobre a área de Broca pode representar uma alternativa viável para pacientes com deficits de nomeação severos.

Palavras-chave: Doença de Parkinson; Afasia de Broca; Estimulação Transcraniana por Corrente Contínua; Teste de Boston para o Diagnóstico das Afasias; Reabilitação

\begin{abstract}
Parkinson's disease (PD) is a neurodegenerative disorder characterized by motor and nonmotor symptoms. PD patients may present language problems, including deficits in confrontation naming. Naming deficits are also an important feature of Broca's aphasia, a condition associated with post-stroke damage to the left inferior prefrontal cortex (Broca's area). We present the case of a 79-year old, male patient diagnosed with both PD (stage 4 in Hoehn and Yahr's scale) and chronic post-stroke, non-fluent aphasia. The patient, with particularly severe naming deficits, was treated with a novel combination of audiovisual production therapyand transcranial direct current stimulation (tDCS), a noninvasive neuromodulatory technique that has been increasingly used to potentiate speech therapy. Anodal tDCS $(2 \mathrm{~mA})$ was applied to the left inferior prefrontal cortex (F7 in the 10/20 system) in nine 20 -min sessions over two weeks while the patient tried to name pictures of common objects aided by short videos of an articulating mouth (audiovisual cue). We found significant pre- to post-training naming improvement for treated items and for untreated, phonemically similar items (generalization). The results provide initial indication that audiovisual production therapy combined with anodal tDCS over Broca's area may represent a viable treatment alternative for patients with severe naming deficits.
\end{abstract}

Keywords: Parkinson Disease; Aphasia, Broca; Transcranial Direct Current Stimulation; Boston Diagnostic Aphasia Examination; Rehabilitation

\footnotetext{
Trabalho realizado no Departamento de Processos Psicológicos Básicos - UnB - Brasília (DF), Brasil.

${ }^{1}$ Programa de Pós-graduação em Ciências do Comportamento, Departamento de Processos Psicológicos Básicos, Instituto de Psicologia - Universidade de Brasília - UnB - Brasília (DF), Brasil.

Conflito de interesses: Não.

Contribuição dos autores: $C A P, R M G R, J S D, M F R L$ e $L G B$ delinearam o estudo; $C A P, R M G R, J S D, M F R L$ e $B A C$ coletaram os dados; todos os autores contribuíram com a análise de dados; $C A P, B A C$ e $L G B$ redigiram o manuscrito.

Financiamento: Coordenação de Aperfeiçoamento de Pessoal de Nível Superior - CAPES-Código de Financiamento 001.

Autor correspondente: Luciano Grüdtner Buratto. E-mail: 1buratto@unb.br

Recebido: Maio 21, 2020; Aceito: Setembro 04, 2020
} 


\section{INTRODUÇÃO}

A doença de Parkinson (DP) é uma doença neurodegenerativa complexa, caracterizada por sintomas motores e não motores. Sintomas motores incluem bradicinesia, tremor de repouso e rigidez muscular. Sintomas não motores podem incluir transtornos psiquiátricos (p.ex., depressão) e cognitivos (p.ex., demência) ${ }^{(1)}$. Problemas de linguagem também são observados em pacientes com DP, incluindo deficit em compreensão de sentenças, em fluência verbal e em nomeação de objetos ${ }^{(2)}$. O prejuízo em nomeação é uma importante característica da afasia de Broca, uma condição associada à lesão pós-acidente vascular cerebral (AVC) no córtex pré-frontal inferior esquerdo ${ }^{(3)}$. Assim, tanto a DP, quanto a afasia de Broca podem, independentemente, prejudicar as habilidades de nomeação e pacientes com ambas as condições podem apresentar deficit de nomeação particularmente severos. Neste estudo, apresentou-se uma evidência inicial de que um tratamento de nomeação em particular (terapia de produção audiovisual), associado à estimulação transcraniana por corrente contínua (ETCC), pode melhorar o desempenho em tarefa de nomeação em um paciente diagnosticado tanto com DP avançada, quanto com afasia pós-AVC severa.

Na terapia de produção audiovisual, a tarefa do paciente é nomear objetos familiares, auxiliado por pistas com quantidades crescentes de informações fonêmicas e visuais sobre as palavrasalvos ${ }^{(4)}$. A nomeação de objetos é facilitada quando o paciente vê um falante dizendo em voz alta o nome do objeto, comparada a quando o paciente somente ouve o falante ${ }^{(4,5)}$. A terapia de produção audiovisual tira proveito da estreita relação entre a produção e a percepção audiovisual da fala ${ }^{(6)}$.A percepção de movimentos da boca relacionados à fala engaja áreas cerebrais essenciais para a percepção e para a produção da fala ${ }^{(7)}$.

Em coerência com essa visão, foi demonstrado que a terapia perceptual audiovisual (relacionada à terapia de produção audiovisual), que envolve o pareamento de nomes apresentados auditivamente com imagens apresentadas visualmente, também pode melhorar a nomeação subsequente para palavras treinadas e não treinadas em pacientes com afasia ${ }^{(8)}$. Essa melhora de nomeação relacionada à terapia pode ser potencializada quando a ETCC é aplicada simultaneamente no córtex pré-frontal esquerdo $^{(9)}$.

A ETCC tem sido extensivamente investigada em ensaios clínicos, incluindo ensaios com pacientes com DP e com afasia pós-AVC ${ }^{(10)}$. Consiste em uma técnica de neuromodulação, que se baseia na estimulação do tecido cortical com uma corrente elétrica gerada por dois eletrodos posicionados sobre o couro cabeludo. Um eletrodo positivo (ânodo) aumenta a excitabilidade cortical, enquanto um eletrodo negativo (cátodo) a diminui. Em estudos sobre a DP, o ânodo tem sido tradicionalmente posicionado sobre M1 (córtex motor) para tratar sintomas motores, e sobre F3 (córtex pré-frontal dorsolateral esquerdo no Sistema Internacional 10/20) para tratar sintomas não motores (p.ex., memória de trabalho). Em estudos sobre a afasia, o ânodo tem sido comumente colocado sobre F7 (córtex pré-frontal inferior esquerdo; área de Broca) para estimular áreas corticais residuais.

Apesar da natureza distribuída das redes cerebrais envolvidas na produção da fala (p.ex., área de Broca, ínsula anterior esquerda, putâmen esquerdo, área motora suplementar), várias linhas de evidência apontam para a área de Broca (F7) como um local de estimulação particularmente promissor em pacientes com afasia. $\mathrm{O}$ aumento da ativação em áreas preservadas do córtex frontal esquerdo está associado com a acurácia de nomeação em pacientes com afasia, sugerindo que o córtex perilesional preservado pode desempenhar um importante papel na recuperação da afasia ${ }^{(11)}$. Além disso, um tratamento similar à terapia de produção audiovisual (speech entrainment) melhorou a produção da fala e aumentou a ativação em áreas preservadas no córtex frontal inferior esquerdo em pacientes com afasia de Broca, sugerindo que o mecanismo de recuperação nessa classe de terapias envolve o recrutamento de áreas perilesionais do córtex frontal esquerdo ${ }^{(5)}$. Esses resultados sugerem que a estimulação da área de Broca (F7) e de tecidos vizinhos podem fornecer uma estratégia terapêutica efetiva para pacientes com afasia de Broca. Aqui, a ETCC foi aplicada sobre F7, enquanto o paciente realizava um treinamento de produção audiovisual conhecido por engajar a área de Broca.

A terapia de produção audiovisual tem mostrado melhorias no desempenho de nomeação em pacientes com afasia ${ }^{(4,5)}$. De maneira similar, a intimamente relacionada terapia perceptual audiovisual, quando associada à ETCC, também tem mostrado melhorias no desempenho de nomeação de pacientes com afasia ${ }^{(9)}$. Contudo, nenhum estudo prévio relatou a aplicação concorrente de terapia de produção audiovisual com ETCC. Assim, o primeiro objetivo deste estudo foi apresentar evidência preliminar sobre a viabilidade dessa nova combinação de tratamentos. Além disso, o protocolo aqui apresentado foi testado em um paciente com DP e afasia crônica. O foco do tratamento foram os deficits de nomeação do paciente, não os prejuízos motores relacionados à DP. O caso é de interesse porque, tanto a DP, quanto a afasia, podem afetar aspectos complementares da fala, resultando em deficits particularmente severos, que podem se mostrar resistentes às terapias fonoaudiológicas tradicionais. Assim, o segundo objetivo deste estudo foi avaliar o potencial benefício desse novo tratamento na acurácia de nomeação de um paciente com prejuízo severo de nomeação (DP e afasia).

\section{APRESENTAÇÃO DO CASO CLÍNICO}

\section{História do caso}

Um homem de 79 anos, destro, com ensino superior completo e sem histórico psiquiátrico prévio participou do estudo. O paciente apresentava DP no estágio 4, conforme a escala de Hoehn e Yahr, bem como um AVC isquêmico e crônico (oito meses) na artéria cerebral média esquerda, confirmado por meio de um exame de ressonância magnética (encefalomalácia córtico-subcortical frontotemporal esquerda). De acordo com sua esposa, o paciente trabalhou como engenheiro civil e era capaz de se comunicar oralmente antes do AVC. Depois do AVC, desenvolveu afasia não fluente severa. A natureza não fluente da afasia foi avaliada por meio de um conjunto de subtestes (p.ex., tarefas de discriminação de palavras, de comandos, de nomeação por confrontação visual e de fluência de nomeação) do Teste de Boston para o Diagnóstico das Afasias (TBDA) (Tabela 1). A classificação de afasia de Broca foi determinada por meio dos critérios de Helm-Estabrooks, Albert e Nicholas ${ }^{(12)}$, uma vez que o paciente mostrou compreensão auditiva relativamente preservada (baseada nos escores do subteste do TBDA), mas repetição severamente prejudicada (baseada nas tarefas de repetição de palavras e de sentenças do protocolo de apraxia verbal de Martins e Ortiz). O paciente fazia uso de 
Tabela 1. Características do paciente e resultados da avaliação inicial

\begin{tabular}{|c|c|}
\hline Variável & Escore \\
\hline Idade (anos) & 79 \\
\hline Sexo & Masculino \\
\hline Educação (anos) & 18 \\
\hline Tempo pós-AVC (meses) & 8 \\
\hline MEEM & 14 \\
\hline \multicolumn{2}{|l|}{ Subtestes do TBDA } \\
\hline Fluência (\%) & 0 \\
\hline Linha melódica (1-7) & 1 \\
\hline Comprimento de sentença (1-7) & 1 \\
\hline Agilidade articulatória (1-7) & 1 \\
\hline Forma gramatical (1-7) & 1 \\
\hline $\begin{array}{l}\text { Parafasia na execução da } \\
\text { repetição da fala (1-7) }\end{array}$ & 1 \\
\hline Busca da palavra (1-7) & 1 \\
\hline Compreensão auditiva (\%) & 43 \\
\hline $\begin{array}{l}\text { Discriminação de palavra } \\
(0-72)\end{array}$ & 35 \\
\hline Comandos (0-15) & 5 \\
\hline Nomeação (\%) & 0 \\
\hline $\begin{array}{l}\text { Nomeação por confrontação } \\
\text { visual }\end{array}$ & 0 \\
\hline Escore C $(0-100)$ & 20 \\
\hline Severidade da afasia $(0-5)$ & 1 \\
\hline Tipo de afasia & Não fluente \\
\hline Apraxia não verbal (0-200) & 80 (leve) \\
\hline Apraxia verbal & $\begin{array}{l}\text { Paciente não conseguia } \\
\text { repetir palavras ou sentenças }\end{array}$ \\
\hline Disartria & $\begin{array}{l}\text { Alterações da ressonância, } \\
\text { da articulação e da prosódia }\end{array}$ \\
\hline $\begin{array}{l}\text { Extensão da lesão (cortical e } \\
\text { subcortical) }\end{array}$ & $\begin{array}{l}\text { Lesão frontal, temporal e } \\
\text { parietal esquerda, incluindo o } \\
\text { giro frontal inferior esquerdo }\end{array}$ \\
\hline
\end{tabular}

Notas: Número entre parênteses representa a amplitude dos escores da escala; Escore $\mathrm{C}=$ escores menores indicam desempenho geral pior; Severidade da afasia = escore menor indica afasia mais severa; Apraxia verbal e não verbal foram avaliadas com o protocolo proposto por Martins e Ortiz

Legenda: $\mathrm{MEEM}=$ Mini Exame do Estado Mental; TBDA = Teste de Boston para o Diagnóstico das Afasias

medicamentos (levodopa e cloridrato de donepezila) durante todo o período de coleta de dados. O cloridrato de donepezila foi introduzido para tratar problemas de memória, conforme relatado por sua esposa; a introdução desse medicamento ocorreu seis meses antes do presente estudo. $\mathrm{O}$ paciente não estava clinicamente deprimido, de acordo com seu geriatra. Além disso, não apresentava deficit auditivos (respostas corretas para estímulos tonais abaixo de $70 \mathrm{~dB}$ em $500 \mathrm{~Hz}, 1 \mathrm{kHz}$, $2 \mathrm{kHz}$ e $4 \mathrm{kHz}$ ), visuais (respostas corretas além da terceira linha da escala de Snellen), ou prejuízos cognitivos severos (14 pontos no Mini Exame do Estado Mental, principalmente devido aos deficits de comunicação oral e escrita; p.ex., ele foi capaz de apontar para objetos-alvo, mas não foi capaz de nomeá-los). Contudo, o paciente apresentou sintomas de fala relacionados à DP, tais como voz suave, fala lenta e fluência verbal reduzida. Importante ressaltar que apresentou apraxia não verbal leve (escore igual a 80 no protocolo de apraxia não verbal proposto por Martins e Ortiz ${ }^{(13)}$ ) e disartria (presença de alterações da ressonância, da articulação e da prosódia). O paciente foi submetido à reabilitação física antes do estudo, mas não à terapia fonoaudiológica. $\mathrm{O}$ estudo foi aprovado pelo Comitê de Ética em Pesquisa com Seres Humanos da Faculdade de Ciências da Saúde da Universidade de Brasília (CAAE: 57633316.0.0000.0030). A cuidadora do paciente assinou o Termo de Consentimento Livre e Esclarecido e autorizou o uso dos registros de som das sessões de teste.

\section{Procedimento}

O experimento ocorreu em dez sessões, realizadas ao longo de duas semanas. A avaliação do paciente foi conduzida na primeira sessão e o tratamento ocorreu nas nove sessões subsequentes. O número de sessões foi escolhido com base em achados de que a nomeação de imagens pode ser melhorada com, pelo menos, cinco sessões de terapia perceptual audiovisual (com ou sem ETCC ${ }^{(8,9)}$ e com até 20 sessões de terapia de produção audiovisual (sem ETCC) ${ }^{(4)}$. Na primeira sessão, o paciente foi avaliado por uma fonoaudióloga treinada (CAP) após encaminhamento feito por neurologista. No fim da primeira sessão, o paciente fez um teste pré-tratamento, com duração de $20 \mathrm{~min}$, em que foram apresentadas 36 imagens e foi solicitado a ele que nomeasse o objeto apresentado em cada imagem. As imagens foram apresentadas por $20 \mathrm{~s}$, com um intervalo entre tentativas de $2 \mathrm{~s}$. As verbalizações do participante foram gravadas para posterior pontuação por juízes. As imagens consistiram em desenhos em preto e branco de objetos comuns, selecionadas de um conjunto de dados amplamente utilizado ${ }^{(14)}$. Os objetos nas imagens correspondiam a palavras monossilábicas, dissilábicas e trissilábicas do português brasileiro, com baixa variabilidade de nomeação. Das 36 imagens, 18 foram posteriormente treinadas nas sessões de treinamento (lista treinada; p.ex., "carro") e 18 não foram treinadas, mas compartilhavam os primeiros fonemas com palavras correspondentes na lista treinada (lista similar; p.ex., "casa") (Figura 1a). A lista similar foi incluída no estudo para avaliar se os potenciais efeitos da terapia audiovisual associada à ETCC seriam generalizados para itens não treinados, mas similares. Os itens treinados e similares foram apresentados em ordem aleatória.

O paciente recebeu nove sessões de 20 min de ETCC e terapia audiovisual combinadas, sendo quatro sessões na primeira semana (de terça a sexta-feira) e cinco na segunda semana (de segunda a sexta-feira). A estimulação foi feita por meio de um aparelho estimulador por corrente contínua, movido a bateria (DC-STIMULATOR, neuroConn GmbH, Ilmenau, Alemanha), consistindo em dois eletrodos envoltos por esponjas embebidas em solução salina $\left(35 \mathrm{~cm}^{2}\right)$. Para estimular a área de Broca (BA $44,45)$, o ânodo foi colocado sobre F7, no hemisfério cerebral esquerdo, de acordo com o Sistema 10/20 do Eletroencefalograma (Figura 1b). O cátodo foi colocado sobre a área supraorbital contralateral. Uma corrente constante de $2 \mathrm{~mA}$ foi aplicada por $20 \mathrm{~min}$, seguindo um protocolo usado em estudos prévios com pacientes com afasia de Broca ${ }^{(15)}$. O estimulador foi ligado $1 \mathrm{~min}$ antes do início da tarefa da terapia audiovisual e foi desligado 1 min depois do final da tarefa. A terapia audiovisual consistiu em uma tarefa de nomeação auxiliada por pistas audiovisuais (Figura 1c). Cada uma das 18 imagens pré-testadas (da lista treinada) foi apresentada em uma tela de computador por $60 \mathrm{~s}$. Durante os primeiros $5 \mathrm{~s}$, o paciente foi encorajado a nomear o objeto apresentado. Em seguida, uma pequena tela de vídeo aparecia, mostrando uma boca em movimento. A boca dizia em voz alta o nome do objeto na tela, por quatro vezes, com um intervalo de $2 \mathrm{~s}$ entre as repetições (tempo médio de apresentação do vídeo $=26 \mathrm{~s}$ ). Depois da retirada do vídeo, a imagem 

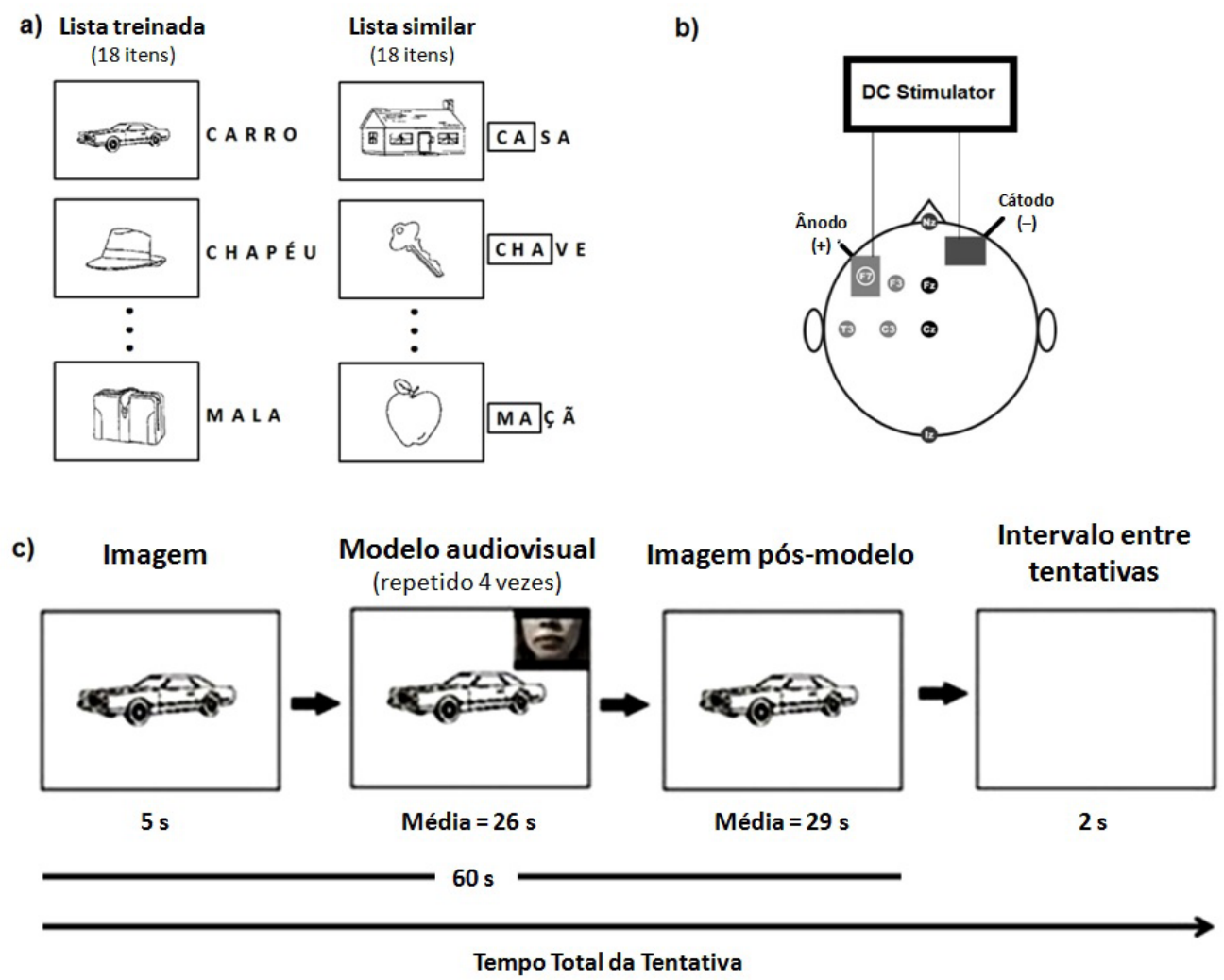

Figura 1. (a) Exemplos de itens treinados e similares. Itens similares não foram treinados, mas compartilhavam os primeiros fonemas com um item treinado correspondente. Itens treinados e não treinados foram avaliados no pré- e pós-tratamento; (b) Representação esquemática da montagem da ETCC; (c) Tentativa durante o tratamento audiovisual e ETCC simultânea

permanecia na tela pelo tempo remanescente (média $=29 \mathrm{~s})$. O participante foi encorajado a nomear o objeto em qualquer momento, durante a tentativa, e a imitar a boca em movimento quando ela estava na tela. A lista de treino foi repetida ao longo das sessões, com diferentes sequências das imagens sendo usadas em cada sessão. Ao final da última sessão, 5 min depois que o estimulador foi desligado, o paciente realizou um teste pós-tratamento de $20 \mathrm{~min}$. O teste pós-tratamento foi idêntico ao teste pré-tratamento, exceto que a sequência de imagens foi novamente aleatorizada. A adesão e a tolerância ao tratamento foram altas, ambas, pois o paciente compareceu a todas as sessões nos horários esperados e não relatou desconfortos maiores. De acordo com sua esposa, o humor dele melhorou acentuadamente ao longo do tratamento. O paciente teve dificuldades de compartilhar sua própria perspectiva, devido aos seus severos deficits de linguagem.

\section{Pontuação}

O desempenho de nomeação foi pontuado com uma versão modificada do sistema de pontuação do Índice Porch de Capacidade Comunicativa ${ }^{(4)}$, que é sensível a pequenas mudanças na capacidade de nomeação. No sistema de pontuação original, a escala varia de 1 (nenhuma resposta) a 16 (resposta acurada sem pistas ou atraso). Na versão modificada aqui usada, a escala variou de 0 (nenhuma resposta) a 9 (nome-alvo correto e produzido sem pistas ou atraso). Escores intermediários representam níveis crescentes de similaridade entre a resposta verbal e a palavra-alvo (1-4 pontos), produção da palavra alvo com apoio de pista (5-6 pontos) ou produção espontânea da palavra alvo (7-9 pontos). A amplitude da escala foi mais curta aqui, comparada a estudos prévios ${ }^{(4)}$, porque o protocolo do estudo não envolveu a apresentação de pistas hierárquicas (p.ex., o sistema de pontuação não tem um escore para "resposta correta quando o primeiro fonema do alvo é dado como uma pista" porque o primeiro fonema não foi fornecido como pista). A Tabela 2 apresenta a versão adaptada do sistema de pontuação do Índice Porch de Capacidade Comunicativa. As gravações com as verbalizações do paciente foram transcritas e avaliadas independentemente, por quatro juízes (RMGR, JSD, MFRL e BAC). A fidedignidade entre juízes das avaliações das respostas do pré e do pós-tratamento foi avaliada por meio do coeficiente de correlação intraclasse, usando uma definição de absoluta concordância, modelo two-way random, $r=0,99$ [0,98, 0,99]. Dada a alta fidedignidade entre juízes, os escores de cada item foram computados como sendo a média de avaliação de todos os juízes naquele item.

\section{RESULTADOS}

Testes $t$ para grupos pareados (pré vs. pós-tratamento) para cada lista, baseados nos escores das palavras individuais, revelaram melhora em nomeação significativa, tanto para as palavras treinadas, $t(17)=2,34, p=0,03$ (bicaudal), quanto para as palavras similares não treinadas, $t(17)=3,95$, $p=0,001$ (bicaudal). Para as palavras treinadas, os escores de 
Tabela 2. Sistema de pontuação do Índice Porch de Capacidade Comunicativa (versão adaptada)

\begin{tabular}{|c|c|c|c|}
\hline Escore & Tipo de resposta & Categoria & Descrição e exemplo \\
\hline 9 & Completa & Espontânea & $\begin{array}{l}\text { Fala corretamente a palavra-alvo sem nenhuma pista, dentro de } 3 \text { segundos (p.ex., } \\
\text { "gato" para o alvo "gato") }\end{array}$ \\
\hline 8 & Erro fonêmico & Espontânea & $\begin{array}{l}\text { Produz fonemas incorretos, mas espontaneamente corrigidos (p.ex., "cato, gato" } \\
\text { para o alvo "gato") }\end{array}$ \\
\hline 7 & Autocorreção & Espontânea & $\begin{array}{l}\text { Responde com uma palavra errada e logo a corrige (p.ex., "cachorro, gato" para o } \\
\text { alvo "gato") }\end{array}$ \\
\hline 6 & Apresentação repetida & Modelação & $\begin{array}{l}\text { Corretamente produz a palavra- alvo pelo menos } 3 \text { segundos depois de assistir } \\
\text { à pista audiovisual (p.ex., diz "dedo" } 3 \text { segundos depois de assistir ao modelo } \\
\text { audiovisual para o alvo "dedo") }\end{array}$ \\
\hline 5 & Produção simultânea & Modelação & $\begin{array}{l}\text { Corretamente produz a palavra-alvo durante a apresentação das pistas } \\
\text { audiovisuais (p.ex., diz "dedo" durante a apresentação da boca modelo } \\
\text { verbalizando "dedo") }\end{array}$ \\
\hline 4 & Incompleta & Relacionada & $\begin{array}{l}\text { Produz uma aproximação, mas não pode produzir completamente a palavra (p.ex., } \\
\text { "cado" para o alvo "gato") }\end{array}$ \\
\hline 3 & Similaridade semântica & Relacionada & $\begin{array}{l}\text { Produz palavra semanticamente relacionada ao alvo (p.ex., "tigre" para o alvo } \\
\text { "gato") }\end{array}$ \\
\hline 2 & Incorreta & Intenção comunicativa & $\begin{array}{l}\text { Não produz quaisquer dos fonemas em uma palavra-alvo, mas produz palavras } \\
\text { não relacionadas ou não palavras (p.ex., "bola" e "teaz" para o alvo "gato") }\end{array}$ \\
\hline 1 & Sem resposta & Intenção comunicativa & $\begin{array}{l}\text { Produz resposta não relacionada, como jargão ou estereotipia (p.ex., "bustiganda" } \\
\text { para o alvo "gato") }\end{array}$ \\
\hline 0 & Sem resposta & Não produzida & $\begin{array}{l}\text { Não produz qualquer resposta ou produz sons não relacionados (e.g., "eeeh" para } \\
\text { "gato") }\end{array}$ \\
\hline
\end{tabular}

Nota: Escores 5 e 6 não se aplicam às avaliações do pré- e pós-tratamento, pois eles se referem apenas à terapia audiovisual, quando foram apresentados os modelos audiovisuais

Tabela 3. Índice Porch de Capacidade Comunicativa em tarefa de nomeação de imagem

\begin{tabular}{|c|c|c|c|c|c|c|c|}
\hline \multirow{2}{*}{ Tipo de palavra } & \multicolumn{2}{|c|}{ Pré-tratamento } & \multicolumn{2}{|c|}{ Pós-tratamento } & \multirow{2}{*}{$t$} & \multirow{2}{*}{$p$} & \multirow{2}{*}{$d$} \\
\hline & $M$ & $D P$ & $M$ & $D P$ & & & \\
\hline Treinada & 1,39 & 0,94 & 2,38 & 1,66 & 2,34 & 0,032 & 0,55 \\
\hline Similar & 1,11 & 0,89 & 1,94 & 0,51 & 3,95 & 0,001 & 0,93 \\
\hline
\end{tabular}

Nota: Similar = palavras não treinadas, mas foneticamente similares; $d=d$ de Cohen, medida de tamanho de efeito

Legenda: $\mathrm{M}=$ Média; $\mathrm{DP}=$ Desvio Padrão; $\mathrm{t}=$ teste $\mathrm{t}$ pareado; $\mathrm{p}=$ valor $\mathrm{p} ; \boldsymbol{d}=d$ de Cohen, medida de tamanho de efeito

nomeação médios aumentaram de 1,39 (pré-tratamento) para 2,38 (pós-tratamento). Para as palavras não treinadas, os escores aumentaram de 1,11 (pré-tratamento) para 1,94 (pós-tratamento). A maioria das respostas consistiu em não palavras, algumas das quais continham fonemas pertencentes à palavra-alvo. Uma das poucas palavras-alvo produzidas corretamente, a partir da apresentação de sua imagem, foi "taça". A Tabela 3 sintetiza os resultados.

Inspeção adicional dos escores do paciente indicou que a melhora em nomeação para as listas treinada e não treinada pode ser atribuível a um aumento na intenção comunicativa. Isso ocorre porque o número de respostas com escore 2 (que indica produção de fonemas não alvos) aumentou de 33,33\% para $90,28 \%$ (lista treinada) e de $45,83 \%$ para $91,67 \%$ (lista similar). Além disso, o número de itens pontuados com 0 (nenhuma resposta) ou 1 (verbalizações estereotipadas) caiu de $56,95 \%$ para $2,78 \%$ (lista treinada) e de $54,16 \%$ para $5,56 \%$ (lista similar). Finalmente, o número de respostas com o escore máximo de 9 (p.ex., corretamente dizer/tasa/ para "taça") aumentou de $0 \%$ para $5,56 \%$ na lista treinada, mas permaneceu em $0 \%$ na lista similar. Em contrapartida, o número de aproximações (escore 4; quando o paciente verbaliza respostas incorretas, mas fonologicamente relacionadas ao alvo; p.ex., /cado/ para "gato") aumentou de 0\% para 2,78\% na lista similar, e caiu de $2,78 \%$ para $0 \%$ na lista treinada.

\section{DISCUSSÃO}

Até onde se sabe, este é o primeiro estudo a avaliar o benefício potencial de terapia de fala audiovisual simultaneamente à estimulação transcraniana por corrente contínua sobre a área de Broca em um paciente com doença de Parkinson (estágio 4) e afasia de Broca não fluente e crônica pós-AVC. Ambas as condições podem prejudicar as habilidades de nomeação ${ }^{(2,3)} \mathrm{e}$, assim, pacientes com ambas as condições podem sofrer deficits de nomeação severos. De fato, o paciente deste estudo obteve a pontuação 0 no componente de nomeação do teste de avaliação. Assim, qualquer melhora no desempenho de nomeação seria notável, neste caso. Observou-se que a capacidade do paciente de nomear objetos visuais comuns melhorou depois do tratamento, o que foi observado tanto para itens praticados, quanto para itens não praticados. Os resultados foram promissores na medida em que o prognóstico para melhora da fala em pacientes com essa combinação, em particular, é muito ruim. Os resultados também forneceram evidência inicial de que a aplicação simultânea de duas técnicas de intervenção orientadas teoricamente - terapia de fala audiovisual ${ }^{(4,5)}$ e ETCC sobre a área de Broca $^{(8,9)}$ - pode melhorar a capacidade de nomeação em um paciente crônico e, portanto, justificam pesquisas adicionais.

A magnitude da melhoria de nomeação foi relativamente pequena. Embora o comportamento de nomeação do paciente tenha mudado de produzir, predominantemente, "nenhuma resposta" para produzir palavras-alvo corretas, houve poucas 
respostas corretas. O sistema de pontuação modificado do Índice Porch de Capacidade Comunicativa ${ }^{(4)}$ aqui usado foi capaz de capturar pequenas mudanças na produção da fala. Se uma escala de resposta binária tivesse sido usada (p.ex., proporção de respostas corretas), seriam observados efeitos piso nos testes de pré-tratamento e de pós-tratamento. A melhora de nomeação foi parcialmente atribuída a um aumento na intenção comunicativa (poucos casos de "nenhuma resposta" e verbalizações estereotipadas, com respectivo aumento da produção de fonemas não alvo). Assim, o tratamento parece não afetar somente processos relacionados à fala (p.ex., mais aproximações e respostas corretas), mas também processos motivacionais e não relacionados à fala (p.ex., menos hesitações e mais tentativas de nomeação por parte do paciente).

Houve várias limitações no presente estudo. Primeiramente, é um estudo de caso, em que uma intervenção fonoaudiológica foi associada com a ETCC em um paciente com DP e afasia de Broca. Dado o número de variáveis envolvidas, é difícil determinar qual fator foi responsável pela melhora de nomeação observada (terapia vs. ETCC vs. ambas) e qual condição médica foi mais sensível ao tratamento (DP vs. afasia de Broca vs. ambas). É possível que a própria terapia de nomeação, sem a ETCC, tenha sido responsável pelo benefício de nomeação. Além disso, é possível, embora improvável, que a melhora de nomeação observada ocorreria naturalmente, sem o tratamento. Ensaios clínicos randomizados permitiriam separar as contribuições únicas da terapia de produção audiovisual e da ETCC no desempenho de nomeação em grupos separados de pacientes com DP (sem AVC no córtex frontal esquerdo), de pacientes com afasia de Broca (sem DP) e de pacientes com ambas as condições.

Em segundo lugar, existem outras razões possíveis para a capacidade de nomeação severamente comprometida do paciente. Deficits de memória, por exemplo, poderiam explicar, parcialmente, o prejuízo de nomeação. O escore do paciente no Mini Exame do Estado Mental foi baixo para sua idade e nível educacional. Além disso, ele fazia uso de cloridrato de donepezila durante a testagem, indicando problemas de memória pré-existentes. Notou-se, contudo, que os escores no Mini Exame do Estado Mental foram mais afetados por suas capacidades de comunicação e, assim, não diretamente imputáveis aos deficits de memória. O paciente seguiu as instruções corretamente, apontando para os objetos-alvo quando solicitado, mas não foi capaz de nomear aqueles objetos quando solicitado a fazê-lo, o que diminuiu consideravelmente seus escores no teste. Embora o Mini Exame do Estado Mental não seja indicado para pacientes não fluentes, ele foi usado aqui principalmente como uma ferramenta de triagem (p.ex., o Mini Mental permitiu checar se o paciente era capaz de seguir instruções simples). Prejuízos motores (p.ex., disartria) e deficits de planejamento e sequenciamento motor (p.ex., apraxia não verbal) provavelmente também não foram as razões principais para os prejuízos de nomeação do paciente. O foco do tratamento foi em nomeação, em vez de exercícios motores orais, estes últimos tradicionalmente usados para fortalecer a musculatura dos orgãos fonoarticulatórios. Embora as sessões de tratamento representem uma oportunidade para exercício motor oral, foi a natureza comunicativa da melhora do paciente que se destacou nas comparações entre pré e póstratamento (p.ex., mais tentativas de nomeação e aumento da produção de fonemas-alvo e similares ao alvo, em oposição a fonemas não relacionados ao alvo). Assim, embora deficits de memória, motores e de planejamento/sequenciamento possam ter afetado as capacidades de nomeação do paciente, provavelmente não constituem a razão principal para a severidade de seus prejuízos de nomeação.

Uma terceira limitação do estudo diz respeito à manipulação da generalização. Metade dos itens de teste foi repetidamente testada durante as sessões de prática (itens treinados); a outra metade não foi treinada (itens não treinados). Tanto itens treinados quanto itens não treinados se beneficiaram do tratamento ${ }^{(9)}$. O que eles tinham em comum eram os primeiros fonemas (p.ex., "carro" e "casa"). Assim, é possível que os itens não treinados tenham se beneficiado do treinado devido à prática do fonema que eles compartilhavam com os itens treinados (p.ex., /ka/). Contudo, como este é um estudo de caso, explicações alternativas são possíveis. Estudo futuro informativo pode incluir um terceiro tipo de item (p.ex., não treinado e sem fonemas compartilhados com um alvo), de modo que, assim, seria possível determinar se o que foi responsável pela melhora de nomeação encontrada para itens não treinados foi a natureza compartilhada de seus fonemas iniciais. Estudos prévios com terapia fonoaudiológica associada à ETCC encontraram resultados mistos a respeito da generalização ${ }^{(8,9)}$. Fridriksson et al. ${ }^{(8)}$ constataram que pacientes tratados com terapia perceptual audiovisual mostraram melhora de nomeação para itens treinados e não treinados, enquanto Baker et al. ${ }^{(9)}$ usaram a mesma terapia e encontraram melhora de nomeação somente para itens treinados. Assim, pesquisas futuras são necessárias para avaliar se os efeitos de terapias fonoaudiológicas associadas à ETCC são generalizáveis para estímulos novos e não treinados. Além disso, os nomes nas listas treinada e não treinada continham uma ampla variedade de fonemas (p.ex., /bo/ em "boca", /de/ em "dedo", /ka/ em "carro"), cujas pronúncias não foram igualmente difíceis para o paciente. Esta é uma limitação, de modo que um conjunto de estímulos personalizado, mais intimamente associado às necessidades linguísticas do paciente, poderia ter produzido melhor resultado.

Finalmente, uma quarta limitação do presente estudo se refere à dosagem do tratamento (o número de sessões foi pequeno) e ao teste de seguimento (que não foi conduzido aqui). A escolha por dez sessões (das quais, nove foram de treinamento) foi motivada por estudos prévios, usando intervenções similares que mostraram melhoras de nomeação significativas ${ }^{(4,9)}$. Estudos futuros com mais sessões podem produzir melhores resultados em pacientes com afasia.

Estudos posteriores também deveriam avaliar o impacto em longo prazo da intervenção. Aqui, a testagem foi conduzida logo após a última sessão de treinamento. Estudos de seguimento, com intervalos mais longos (p.ex., duas semanas, um mês), permitiriam estabelecer se os benefícios do tratamento em nomeação são duradouros.

\section{COMENTÁRIOS FINAIS}

Neste estudo, apresentou-se um tratamento à prova de conceito - terapia de fala audiovisual associada à estimulação transcraniana por corrente contínua sobre a área de Broca - que pode melhorar o desempenho de nomeação em um paciente com deficits de nomeação severos. O desempenho de nomeação melhorou para itens praticados e não praticados em um paciente que apresentava afasia de Broca e Doença de Parkinson. 


\section{AGRADECIMENTOS}

Agradecemos ao IMPI - Instituto de Medicina e Psicologia Integradas - Brasília, Brasil, por permitir acesso a equipamentos e instalações. Também agradecemos ao paciente e a sua esposa pela colaboração neste estudo.

\section{REFERÊNCIAS}

1. Kalia LV, Lang AE. Parkinson's disease. Lancet. 2015;386(9996):896912. http://dx.doi.org/10.1016/S0140-6736(14)61393-3. PMid:25904081.

2. Henry JD, Crawford JR. Verbal fluency deficits in Parkinson's disease: A meta-analysis. J Int Neuropsychol Soc. 2004;10(4):608-22. http:// dx.doi.org/10.1017/S1355617704104141. PMid:15327739.

3. Richardson JD, Fillmore P, Rorden C, LaPointe LL, Fridriksson J. Reestablishing Broca's initial findings. Brain Lang. 2012;123(2):125-30. http://dx.doi.org/10.1016/j.bandl.2012.08.007. PMid:23058844.

4. Choe Y, Stanton K. The effect of visual cues provided by computerised aphasia treatment. Aphasiology. 2011;25(9):983-97. http://dx.doi.org /10.1080/02687038.2011.569893.

5. Fridriksson J, Hubbard HI, Hudspeth SG, Holland AL, Bonilha L, Fromm D, et al. Speech entrainment enables patients with Broca's aphasia to produce fluent speech. Brain. 2012;135(Pt 12):3815-29. http://dx.doi.org/10.1093/brain/aws301. PMid:23250889.

6. van Wassenhove V, Grant KW, Poeppel D. Visual speech speeds up the neural processing of auditory speech. Proc Natl Acad Sci USA. 2005;102(4):1181-6. http://dx.doi.org/10.1073/pnas.0408949102. PMid:15647358.

7. Rorden C, Davis B, George MS, Borckardt J, Fridriksson J. Broca's area is crucial for visual discrimination of speech but not non-speech oral movements. Brain Stimul. 2008;1(4):383-5. http://dx.doi.org/10.1016/j. brs.2008.08.002. PMid:19421338.

8. Fridriksson J, Baker JM, Whiteside J, Eoute D Jr, Moser D, Vesselinov $\mathrm{R}$, et al. Treating visual speech perception to improve speech production in nonfluent aphasia. Stroke. 2009;40(3):853-8. http://dx.doi.org/10.1161/ STROKEAHA.108.532499. PMid:19164782.

9. Baker JM, Rorden C, Fridriksson J. Using transcranial direct-current stimulation to treat stroke patients with aphasia. Stroke. 2010;41(6):1229-36. http://dx.doi.org/10.1161/STROKEAHA.109.576785. PMid:20395612.

10. Lefaucheur JP, Antal A, Ayache SS, Benninger DH, Brunelin J, Cogiamanian F, et al. Evidence-based guidelines on the therapeutic use of transcranial direct current stimulation (tDCS). Clin Neurophysiol. 2017;128(1):56-92. http://dx.doi.org/10.1016/j.clinph.2016.10.087. PMid:27866120.

11. Fridriksson J, Bonilha L, Baker JM, Moser D, Rorden C. Activity in preserved left hemisphere regions predicts anomia severity in aphasia. Cereb Cortex. 2010;20(5):1013-9. http://dx.doi.org/10.1093/cercor/ bhp160. PMid:19687294.

12. Helm-Estabrooks N, Albert ML, Nicholas M. Manual of aphasia and aphasia therapy. 3rd ed. Austin, TX: Pro-Ed; 2014.

13. Martins FC, Ortiz KZ. Proposta de protocolo para avaliação da apraxia da fala. Fono Atual. 2004;7:53-61.

14. Pompeia S, Miranda MC, Bueno OF. A set of 400 pictures standardised for Portuguese: norms for name agreement, familiarity and visual complexity for children and adults. Arq Neuropsiquiatr. 2001;59(2B):330-7. http://dx.doi.org/10.1590/S0004-282X2001000300004. PMid:11460174.

15. Holland R, Crinion J. Can tDCS enhance treatment of aphasia after stroke? Aphasiology. 2012;26(9):1169-91. http://dx.doi.org/10.1080 /02687038.2011.616925. PMid:23060684. 